\title{
Archéopages
}

Archéopages Archéologie et société

46 | 2018

Maisons

\section{Une maison dans tous ses états...}

La résidence seigneuriale d'Ahuy entre le $\mathrm{XI}^{\mathrm{e}}$ et le XVIII ${ }^{\mathrm{e}}$ siècle

A house in all its forms ... The Seigniorial residence of Ahuy between the 11th and 18th centuries

Una vivienda en todos sus estados: la residencia señorial de Ahuy entre los siglos XI y XVIII

\section{Stéphanie Morel Lecornué}

\section{(2) OpenEdition}

Journals

Édition électronique

URL : https://journals.openedition.org/archeopages/4182

DOI : 10.4000/archeopages.4182

ISSN : 2269-9872

Éditeur

INRAP - Institut national de recherches archéologiques préventives

Édition imprimée

Date de publication : 1 décembre 2018

Pagination : 60-69

ISSN : 1622-8545

Référence électronique

Stéphanie Morel Lecornué, « Une maison dans tous ses états... », Archéopages [En ligne], 46 | 2018, mis en ligne le 01 décembre 2020, consulté le 03 juin 2021. URL : http://journals.openedition.org/ archeopages/4182; DOI : https://doi.org/10.4000/archeopages.4182 


\section{Une maison dans}

\section{tous ses états...}

\section{La résidence seigneuriale d'Ahuy entre le $x l^{e}$ et le XVIII siècle}

Stéphanie Morel Lecornué Irrap

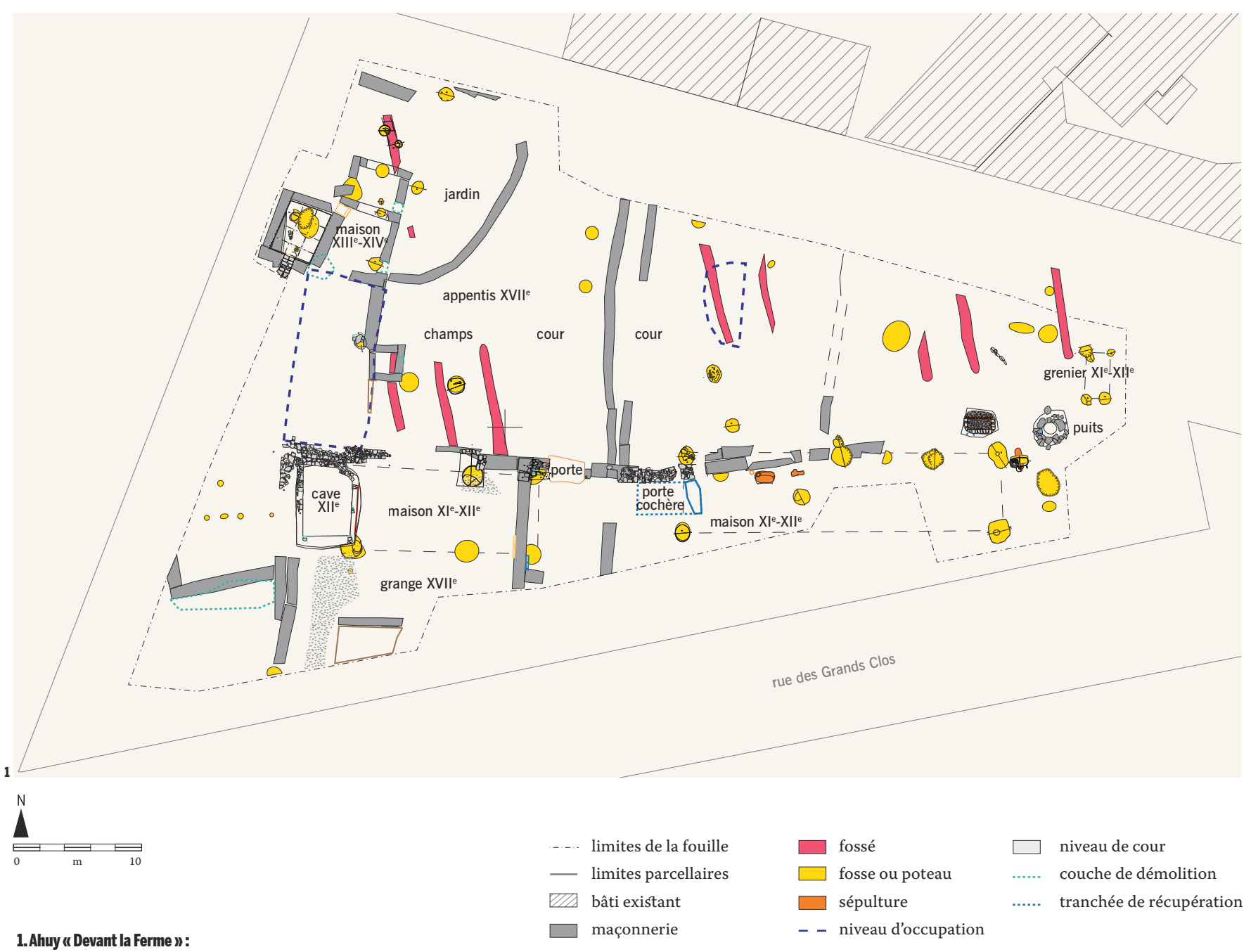


Une fouille réalisée en 2014 par l'Inrap à Ahuy (Côte-d'Or) a mis au jour un habitat privilégié, en bordure sud du village ancien, sur un terrain vierge de toute construction ${ }^{\mathbf{1}}$ (Morel Lecornué, 2015). Le village d'Ahuy est situé dans la vallée du Suzon, à $5 \mathrm{~km}$ au nord-ouest de Dijon; il est construit sur le modèle du village-rue, sur un axe est-ouest. Il est mentionné pour la première fois en 885 dans un diplôme de Charles le Gros, sous le toponyme «Aqueductu » (Arch. dép. Côte-d'Or, G 125, fol. $5 \mathrm{r})$. Les $1800 \mathrm{~m}^{2}$ fouillés ont permis la mise au jour d'un habitat évoluant entre le XI ${ }^{\mathrm{e}}$ siècle et la fin du XVIII ${ }^{e}$ siècle. Plusieurs bâtiments de différentes tailles ont été découverts, dont au moins trois peuvent être désignés avec certitude comme des maisons [ill. 1]. Les sources du XVIII ${ }^{\mathrm{e}}$ siècle permettent par ailleurs d'identifier cet habitat comme la maison seigneuriale d'Ahuy, appartenant aux chanoines de Saint-Étienne de Dijon.

\section{L'habitat des $\mathrm{XI}^{\mathrm{e}}$-XII ${ }^{\mathrm{e}}$ siècles}

La première occupation réelle du site intervient au XI ${ }^{\mathrm{e}}$ siècle : trois sépultures des $\mathrm{VIII}^{\mathrm{e}}$ - $\mathrm{IX}^{\mathrm{e}}$ siècles, mises au jour au sud de l'emprise, semblent isolées; elles indiquent néanmoins la présence d'un habitat proche. Au XI ${ }^{\mathrm{e}}$ siècle sont édifiés deux grands bâtiments sur poteaux porteurs associés à des fossés de culture et à un grenier rectangulaire sur quatre poteaux. Passons vite sur le premier bâtiment, situé à l'est de l'emprise de fouille. Rectangulaire, son ossature repose sur de gros poteaux porteurs. Il n’a livré que très peu de mobilier et aucun niveau de sol. Sa superficie importante $\left(120 \mathrm{~m}^{2}\right)$ peut correspondre à un logement.

Le bâtiment ouest est plus intéressant. Rectangulaire, il comporte une cave, située sous le quart ouest de l'édifice. Le bâtiment sur poteaux associé à cette cave n'a pas été vu lors du premier décapage, car il était sous les murs de l'époque moderne [ill. 2 et 3]. Les murs de cette cave maçonnée ont été en partie récupérés ; seule la partie nord-ouest est bien conservée. Cette pièce excavée de $17 \mathrm{~m}^{2}$ comporte des gradins, insérés dans le mur nord, aménagement interprété comme un soupirail qui descendrait jusqu'au sol de la cave [ill. 4]. L'entrée se situe peut-être à côté de ce puits de lumière. En effet, le mur primitif forme un arrondi dans l'angle nord-est, que rien d'autre ne justifie.

L'ensemble forme donc une maison d'environ $90 \mathrm{~m}^{2}$ au sol, avec des pièces à vivre au premier niveau et une cave ayant probablement fonction de cellier au sous-sol. Le sol de cette cave était situé au minimum à $1 \mathrm{~m}$ sous le sol du bâtiment. Ce bâtiment est daté, grâce à quelques tessons de céramique, du XI ${ }^{\mathrm{e}}$ siècle. Il est possible que le creusement de la cave intervienne dans une seconde étape de construction de la maison, car il semble que le côté oriental de cette pièce enterrée empiète sur une partie des poteaux en bois de l'extrémité occidentale du bâtiment en matériaux périssables. Les relations stratigraphiques ne sont pas évidentes, car cette zone était assez perturbée. De plus, le type de construction ainsi que les rares tessons de céramique ramassés sur le sol de la cave datent celle-ci du XII ${ }^{e}$ siècle. Cette maison a perduré plusieurs siècles, jusqu'à l'érection d'une grange à l'époque moderne reposant sur les murs de la cave et reprenant le tracé de l'habitat primitif. La durée d'utilisation de cette maison semble longue, puisqu'aux XIV ${ }^{e}$-XVII ${ }^{e}$ siècles ses murs ou tout du moins ses fondations étaient encore visibles et propres.

En Bourgogne, à cette période, les maisons étaient souvent mixtes, réunissant sous le même toit hommes et animaux. L'habitat rural est formé de fonds de cabane, de silos, de bâtiments sur poteaux porteurs et de greniers (Saint-Jean Vitus, 2012). Il existe cependant quelques constructions maçonnées à cette période : pour le Moyen Âge central, le seul exemple véritablement fouillé reste celui du Verger au pied de la falaise de SaintRomain (Côte-d'Or) qui montre lévolution d'un petit groupe isolé de bâtiments fréquentés par une population plutôt aisée, au cours des $\mathrm{XI}^{\mathrm{e}}$ $\mathrm{XII}^{\mathrm{e}}$ siècles, puis dans un dernier état au XIV ${ }^{\mathrm{e}}$ siècle.

Des fossés situés au nord des deux bâtiments leur sont associés. Relativement étroits et proches les uns des autres, ils sont parallèles entre eux et implantés selon une trame précise. Ces structures fossoyées sont interprétées comme des vestiges agricoles. L'espacement entre les axes de tranchées est faible $-3,45 \mathrm{~m}$ en moyenne et pourrait correspondre éventuellement à des plantations de vignes.

Pour cette période des $\mathrm{XI}^{\mathrm{e}}$-XII ${ }^{\mathrm{e}}$ siècles, on peut donc imaginer deux grandes maisons rectangulaires, appartenant à des cultivateurs dont les champs sont situés en contrebas de ces bâtiments.

\section{La riche maison du XIII ${ }^{\mathrm{e}}$ siècle}

Le XIII ${ }^{\mathrm{e}}$ siècle marque un tournant dans l'histoire du site. Une nouvelle maison est construite, sur un autre plan et de manière différente. Elle détruit une partie des fossés agricoles précédemment mentionnés, ce qui signale un remaniement du site [ill. 5]. S'il est fort probable que la maison sur cave décrite ci-dessus est encore utilisée lors de l'érection de ce nouvel habitat, il est néanmoins possible que sa destination change et qu'elle devienne une simple grange. Le nouveau bâtiment en L, situé au nordouest de l'emprise de la fouille, comporte trois pièces de tailles équivalentes. Il est maçonné et sa construction est soigneuse. Chaque pièce est munie d'une ou deux ouvertures sur l'extérieur, caractérisées par un seuil de porte ou un escalier. Un muret, conservé sur une seule assise, ceinturait une petite cour située à l'est de la maison. Un dernier mur, mis au jour au sud de ce bâtiment, dans le prolongement du mur est, correspond peut-être à une pièce supplémentaire. Mal de Devant la Ferme menée sous la direction de Stéphanie More

Lecornué, Inrap.

2. In eodem Divionensis pago, villa videlicet Aqueductu, cum capelle. 
conservé, il était pris sous un niveau marneux beige qui a servi de remblai de nivellement à l'époque moderne.

Les deux pièces orientales de cette maison ont été découvertes au moment du diagnostic et ont fourni assez peu d'éléments. La pièce $\mathrm{A}$, la plus grande, comporte un escalier, situé à l'ouest, qui a été bouché depuis l'intérieur de la pièce : le mur est parementé à l'intérieur [ill. 6]. Une porte permet l'accès à la cour/jardin. La pièce $B$, plus petit espace connu de cet habitat, mesure seulement 7,5 $\mathrm{m}^{2}$. Elle est pourvue d'une ouverture sur l'extérieur, dont le seuil, large de o,70 m, est formé de grandes dalles calcaires. La troisième pièce (pièce $C$ ), interprétée comme un cellier semi-enterré, a apporté beaucoup d'informations utiles pour la compréhension de ce bâtiment et du site en général [ill. 7]. De belle facture, cette pièce rectangulaire de $11 \mathrm{~m}^{2}$ comprend un escalier de cinq marches côté sud. Cet escalier a été muré, de l'intérieur de la pièce, ce qui suppose l'existence d'une seconde ouverture, probablement située dans le plafond, non conservé. Deux niveaux de sols successifs ont été mis au jour. Le premier est en terre battue, le second repose sur un radier en hérisson. Un mur de soutènement, postérieur à ce radier, renforce le mur ouest. Adossé au terrain naturel, ce mur a probablement subi une forte poussée, qui a nécessité ce renfort. Le comblement de cette pièce, interprétée comme un cellier, intervient au début du XIV ${ }^{\mathrm{e}}$ siècle. En effet, tout le mobilier retrouvé dans les niveaux de sol, comme dans les remblais, est daté de la fin du XIII ${ }^{\mathrm{e}}$-début du XIV ${ }^{\mathrm{e}}$ siècle. Le comblement de ce cellier ne signifie pas pour autant la fin de l'occupation du reste de la maison, qui a pu perdurer encore quelques dizaines d'années. En effet, dans le comblement supérieur de cette pièce, quelques éléments de céramique des $\mathrm{XVI}^{\mathrm{e}}-\mathrm{XVII}{ }^{\mathrm{e}}$ siècles ont été découverts.

Le mobilier céramique du bas Moyen Âge se concentre presque exclusivement dans la pièce $C$. Ce contexte a livré 1217 tessons. Les récipients reconstitués sont très fragmentés et incomplets, ce qui suggère qu'ils proviennent d'un dépotoir situé en dehors du bâtiment. Le cellier a pu être comblé par une vidange de latrines ou un curage de dépotoir. Les éléments céramiques [ill. 8] sont particulièrement intéressants et forment un lot de qualité : l'éventail des formes est assez limité et a trait majoritairement à la consommation du vin.

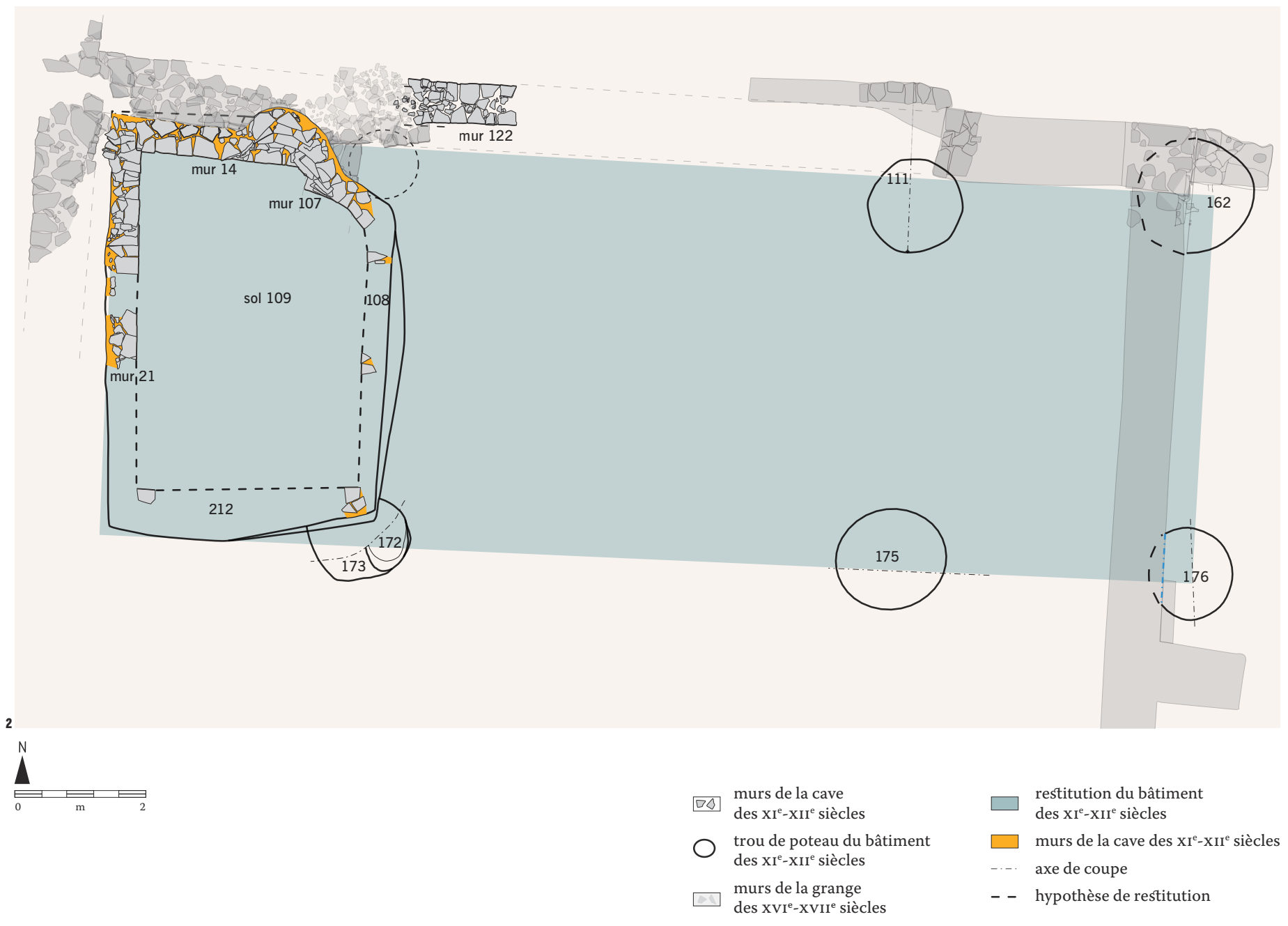




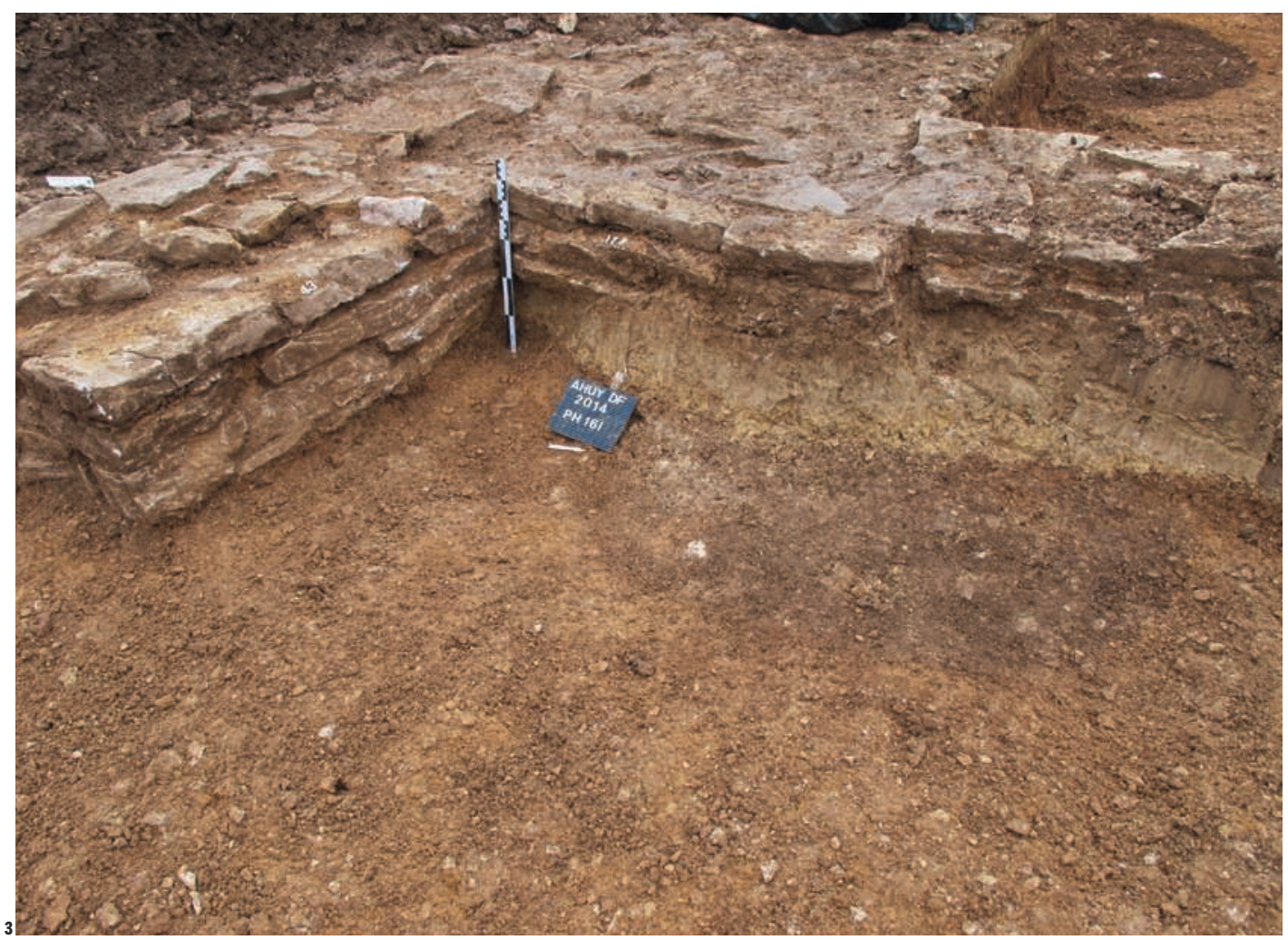

2. Plan général du bâtiment des XI $\left.\right|^{-}$-xl ${ }^{e}$ siècles : une cave et un bâtiment sur poteaux. Seule la partie occidentale est excavée. Les trous de poteau mesurent 1,40 à $1,60 \mathrm{~m}$ de diamètre. Le mur 122 est peut-être un reste des élévations primitives. En grisé,l'édifice d'époque moderne.
3. La fosse 176, appartenant à l'un des bâtiments du xle siècle, se situe sous les murs de la grange d'époque moderne. Entre le mur et la fosse, on distingue une couche marneuse jaune-beige, qui correspond à un remblai de nivellement du xve siècle.

4. La cave des $\mathrm{xl}^{\circ}-\mathrm{xll}^{\circ}$ siècles. On aperçoit le soupirail dans le mur nord. En arrière-plan, les murs du bâtiment de l'époque moderne qui reposent sur ceux de la cave,

$40 \mathrm{~cm}$ plus au nord. Ȧ droite du cliché, on aperçoit une

légère excavation,

correspondant probablement

au trou du poteau nord-ouest de l'édifice.

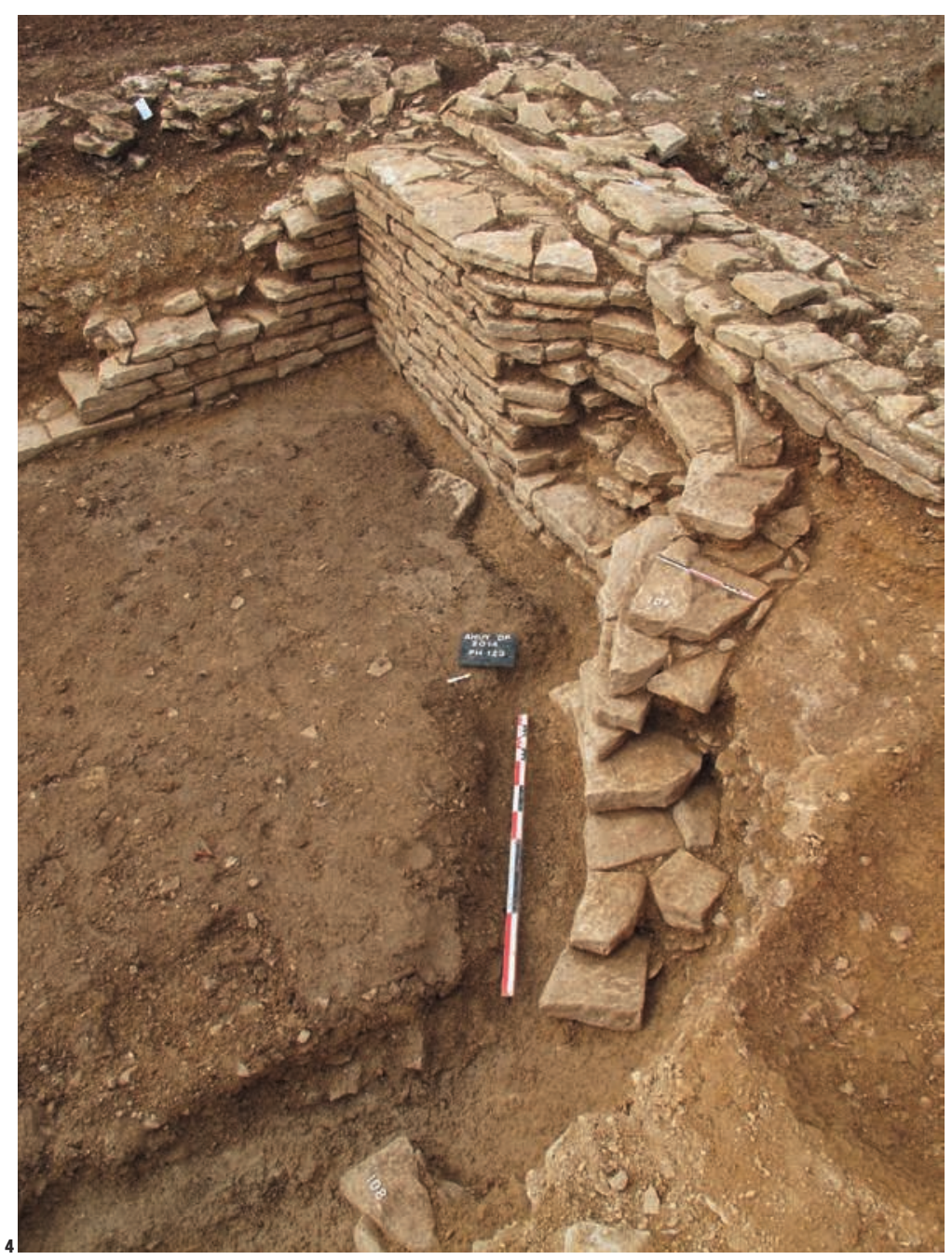

ஜ 


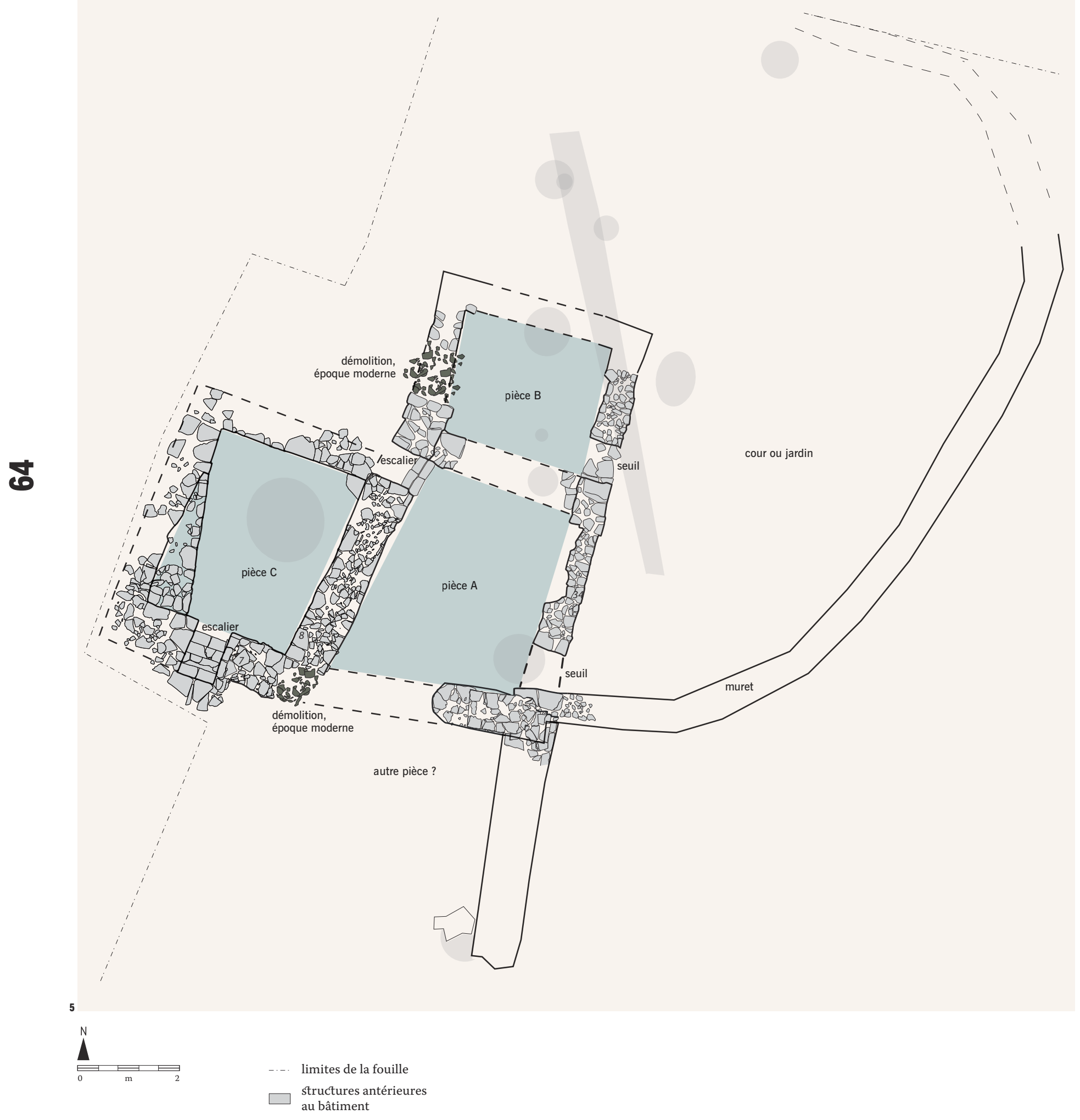

5. Plan général de la maison datée de la fin du xIII' sièclexIve siècle. En grisé, les structures archéologiques antérieures.

6. Maison du XIII'-xIVe siècle, escalier de la pièce A, mure. À droite du cliché, le mur de refend entre les pièces A et $B$, coupé lors du diagnostic.
7. Maison du XIII'-xIVe siècle, radier de la pièce $C$ en cours de fouille. $0 n$ aperçoit

à droite sur le cliché le mur de soutènement, qui repose sur le radier de sol; en arrière-plan, l'escalier qu mène à l'extérieur de la maison. 




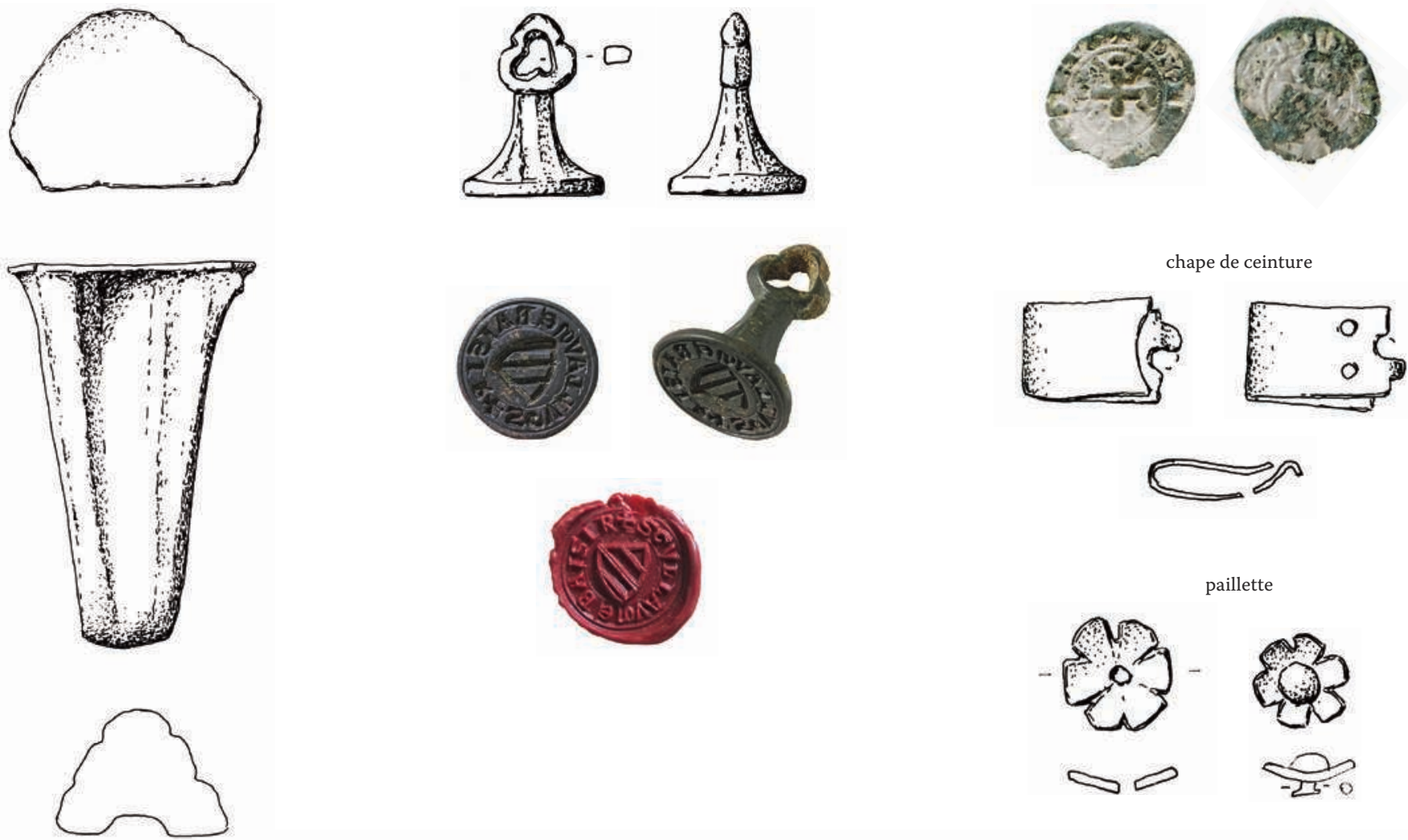

paillette

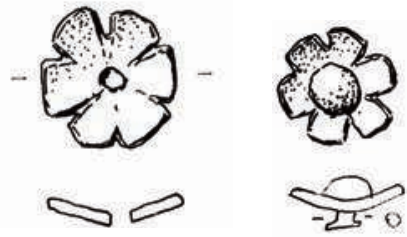

couteau
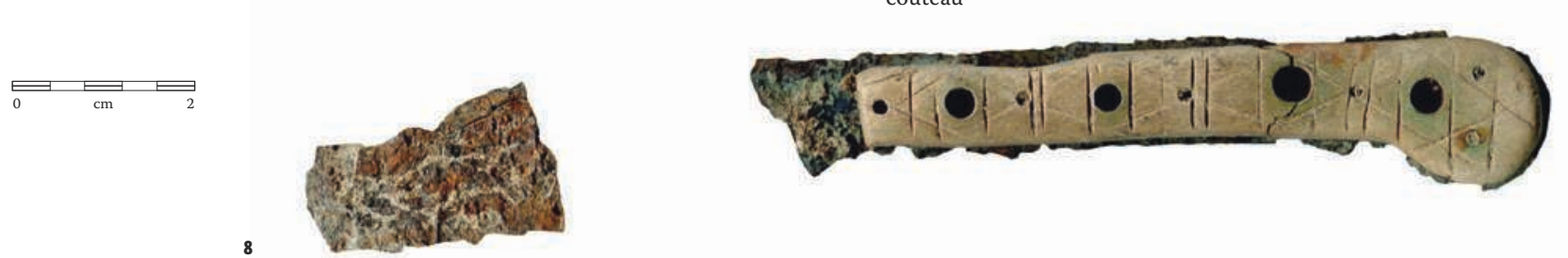

Les vases à liquide (pichets, cruches, gourdes) représentent en effet près de $50 \%$ des récipients découverts dans le remblai de la pièce, voire $75 \%$ si l'on inclut les pots à bec tubulaire. Une forme quasi inconnue de cruche a été mise au jour : il s'agit d'une cruche à deux anses opposées et bec tubulaire rapporté ${ }^{3}$. Ajoutons que la part de la céramique décorée est très importante, ce qui place le site dans un contexte seigneurial comme celui de la ferme de la Grange-du-Mont à Charny (Côte-d'Or), où les pichets glaçurés étaient également très nombreux (Beck, 1989). La présence des pichets de Saintonge à Ahuy signe, comme dans le cas du bâtiment II de Dracy, le statut élevé de ces habitants (Pesez, 1987). Tous ces éléments attestent que l'on est en présence d'une demeure de qualité. Le mobilier métallique confirme la richesse des lieux, par la découverte de paillettes, de chapes de ceinture, d'un pied de chaudron en bronze, etc. ${ }^{\mathbf{4}}$. Une matrice de sceau en alliage cuivreux a également été mise au jour, dans les niveaux de remblais inférieurs [ill. 8]. Cette matrice est ornée du viel écu de Bourgogne, associé au nom de Guillaume Baisir. Le nom de Baisir est inconnu à Ahuy, aussi bien aujourd'hui que dans les archives consultées ; il semble provenir de la région de Namur (Belgique) ${ }^{\mathbf{5}}$. Il pourrait s'agir d'un proche des ducs de Bourgogne (peut-être un écuyer), également proche du propriétaire de cette riche maison, voire du propriétaire lui-même. Enfin, les restes fauniques appuient l'hypothèse d'une maison seigneuriale en présentant un faciès dominé en nombre de restes par les caprinés. D'autres éléments, tels que la consommation de morceaux de choix, d'individus souvent jeunes, ou encore la présence du lapin laissent envisager un statut social élevé .

Les éléments architecturaux, combinés au mobilier découvert dans cet espace, permettent donc de conclure que cet ensemble de petites pièces aux portes étroites et aux escaliers raides correspond à une maison; il ne peut s'agir de granges ni d'étables. Plusieurs textes mentionnant les propriétés des chanoines de Dijon à Ahuy ont été découverts aux Archives départementales de la Côte-d'Or ${ }^{7}$. L'un d'eux date de 1248 et se présente comme un accord, en forme d'échange, par lequel Bertrand Pelerin, chambrier du duc de Bourgogne, cède aux religieux de Saint-Étienne

. Recherches effectuées

Pinguet. 


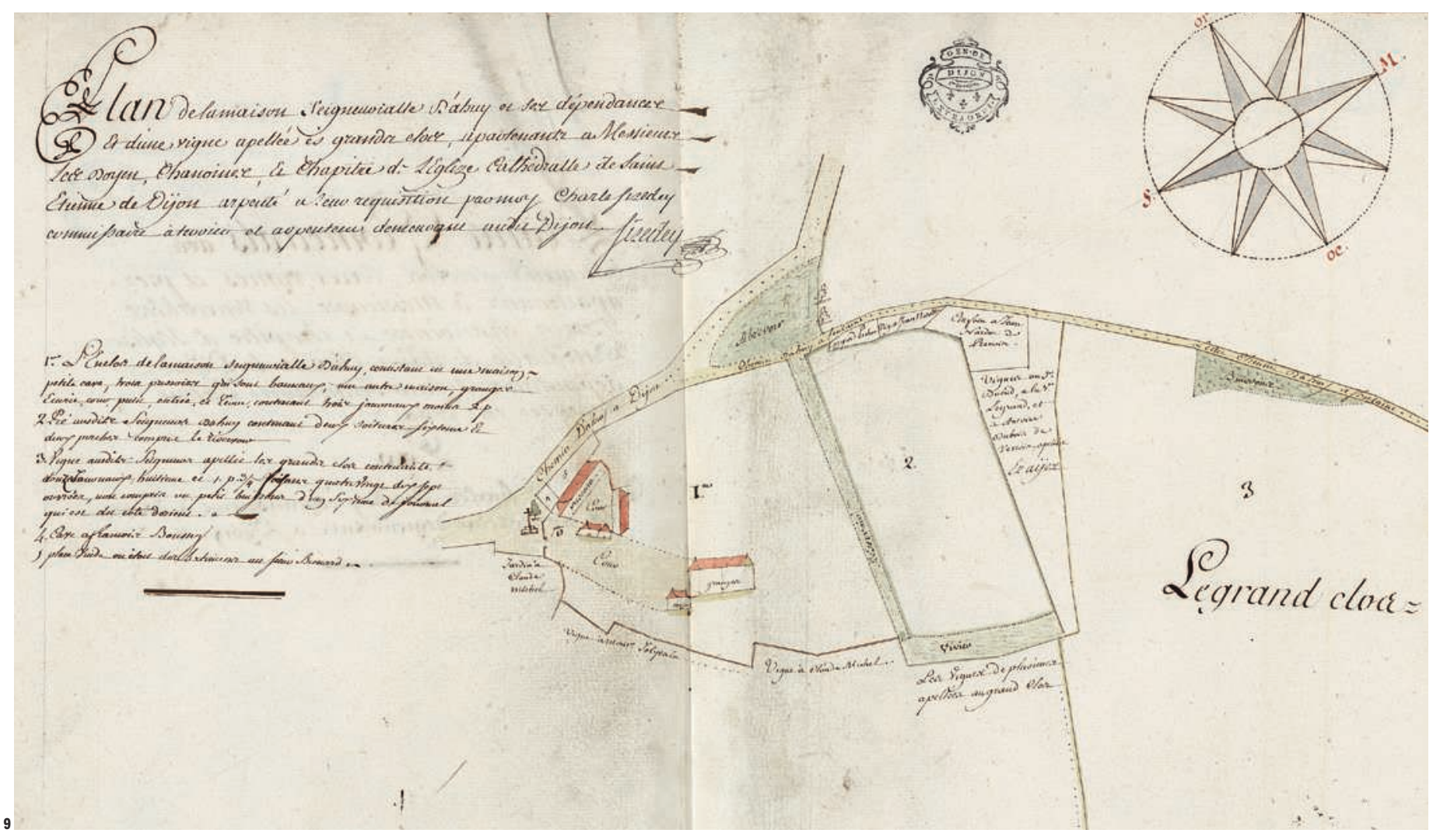

de Dijon tous ses droits ainsi que ceux de Gervais Chauchars, son beau-père, sur le grand enclos situé derrière la grange d'Ahuy, qui appartient à ce monastère (Arch. dép. Côte-d'Or, G 309). Ce texte ne mentionne pas encore de maison seigneuriale.

Cette maison possédait donc plusieurs pièces et probablement un étage. L'absence de tuiles et la présence de nombreuses plaquettes calcaires dans le comblement du cellier plaident pour une couverture en laves, posée sur des murs en pierres sèches (aucune trace de mortier n'a été retrouvée). Plusieurs habitats du bas Moyen Âge ont été fouillés à proximité de ce village. Ainsi de grands bâtiments en pierres, coiffés de laves, de construction soignée, habités aux XIII ${ }^{\mathrm{e}}$-XIV ${ }^{\mathrm{e}}$ siècles, découverts à Saint-Martin-du-Mont, la Vie aux Maires (Chopelain, 2016). On trouve notamment un édifice divisé en quatre pièces. Cet habitat en dur est postérieur à un autre bâtiment sur poteaux. L'évolution de ce site ressemble donc à celui d'Ahuy, tout comme le mobilier retrouvé lors de la fouille.

\section{Une grange à l'époque moderne ?}

Plusieurs bâtiments sont construits à l'époque moderne. L'un d'eux se superpose quasi exactement à la maison du $\mathrm{XI}^{\mathrm{e}}$ siècle, ce qui permet de supposer que le bâtiment primitif était encore en élévation ou, tout du moins, visible [cf. supra ill. 2]. Ce grand bâtiment rectangulaire était composé d'une seule grande pièce de $190 \mathrm{~m}^{2}$. Un mur de refend a été rajouté dans un second temps, créant ainsi une pièce plus petite à l'ouest. Ce bâtiment maçonné peut être interprété de plusieurs façons. Plusieurs éléments font pencher la balance en faveur d'une grange. Les dimensions tout d'abord, assez imposantes, ainsi que la présence d'un dallage au sol. Composé de pierres calcaires plates bien serrées, ce dallage formait un sol dur, qui se rapproche plutôt d'un sol d'écurie. Cependant, le mobilier métallique découvert sur l'ensemble du site contredit cette hypothèse. En effet, très peu d'outils typiquement agro-pastoraux ou viticoles ont été mis au jour, tandis que de nombreux objets de la vie quotidienne apparaissent : objets de piété, de culture, objets personnels, qui laissent envisager que le site a une fonction essentiellement d'habitat. La seconde hypothèse serait donc que le grand bâtiment est une maison, de taille imposante. Il existait peutêtre des subdivisions, qui ont disparu à cause des labours (l'arase des murs était situé à une dizaine de centimètres sous le sol seulement). Au XvI ${ }^{\mathrm{e}}$ siècle, le terme de " grange » désigne une exploitation agricole et non le simple bâtiment de stockage. L'exploitant peut être un métayer dépendant d'un seigneur, ce qui semble être le cas ici.

De nombreux murs ont été découverts à l'est de ce bâtiment. Ces constructions semblent plutôt être des murs de clôture, séparant différentes cours. Le mur est-ouest est ainsi percé de deux grandes portes cochères. La porte orientale est particulièrement bien aménagée : elle est formée d'un passage pour les piétons, associé à une porte cochère. Un petit caniveau était adjoint à ce passage. De nombreux indices démontrent que ces cours ont évolué dans le temps : la porte 
cochère a été murée et certains murs ont été doublés. La cour située au sud a ainsi été divisée en deux (au moins) par l'adjonction d'une cloison.

Les textes concernant la maison seigneuriale d'Ahuy sont plus nombreux et plus descriptifs à partir du $\mathrm{Xv}^{\mathrm{e}}$ siècle. Tout en restant prudent, on peut essayer de mettre en parallèle les vestiges archéologiques et les éléments textuels. Ainsi, en 1451, le Manuel des Censes (Arch. dép. Côte-d'Or, $\mathrm{G} 230$ et $\mathrm{G} 318$, fol. 1) mentionne la maison seigneuriale des chanoines de Saint-Étienne et indique qu'elle possède « une chambre-grenier, deux granges, une écurie, une cour, devant un pré entouré de murailles ». Le texte mentionne également une autre cour avec deux halles abritant trois pressoirs banaux. Le terrier du chapitre de Saint-Étienne de 1452 (Arch. dép. Côte-d'Or, $\mathrm{G}$ 233) donne d'autres précisions. On apprend ainsi que le terrain dénommé le Grand Clos est planté de vignes. Les noms de deux locataires du $\mathrm{XV}^{\mathrm{e}}$ siècle sont connus : Huguenin Motet en 1473, Pierre Millot de Beaulieu en 1482 (Arch. dép. Côte-d'Or, E 2177, fol. 42r-45v et G 233, fol. 1). Les modifications de l'époque moderne, visibles notamment dans les murs de clôture, pourraient être rapprochées du partage en quatre lots équivalents, en 1661, des bâtiments, enclos, vivier et terres du domaine, chacun des lots étant affecté à un chanoine de Saint-Étienne de Dijon (Arch. dép. Côte-d'Or, G 318, fol. 31). Un plan terrier de 1751 figure cette maison seigneuriale [ill. 9], située en lisière du village.

\section{Le statut social des habitants successifs}

L'étroitesse de l'emprise de fouille ne permet d'appréhender qu'une partie des habitations, granges et cours situées aux confins d'Ahuy; les limites de l'habitat, notamment, nous échappent. Néanmoins, les indices sont suffisants pour permettre d'esquisser l'évolution du bâti et par là du statut social des différents propriétaires et locataires.
Les premiers bâtiments des $\mathrm{XI}^{\mathrm{e}}$-XII ${ }^{\mathrm{e}}$ siècles qui peuvent être interprétés comme des maisons, probablement mixtes, relèvent d'un habitat rural, vraisemblablement relativement aisé. Un monde d'agriculteurs, assez riches pour construire une cave maçonnée.

Un changement net intervient au XIII ${ }^{\mathrm{e}}$ siècle, avec l'édification d'une maison en dur, comportant plusieurs pièces et très probablement un étage, ouvrant sur une cour ou un jardinet. Sa construction soignée confirme la richesse de ses propriétaires. Les murs sont maçonnés, le toit est probablement constitué de laves. Les chanoines de Saint-Étienne louent cette maison, vraisemblablement à de riches fermiers. Les nombreuses mentions de pressoirs indiquent que ces exploitants sont probablement des vignerons. À partir de cette période, on peut supposer que les animaux sont séparés des hommes. Ils sont peut-être repoussés dans l'ancienne maison transformée en grange. Les textes confirment ensuite que cette propriété des chanoines de Dijon subit moult évolutions au fil des années (incendie, partage, reconstructions...).

Où se situe la maison seigneuriale à l'époque moderne ? Peut-être au même emplacement qu'au $\mathrm{XIII}^{\mathrm{e}}$ siècle, dans les étages supérieurs disparus, au-dessus du cellier condamné au début du $\mathrm{XIV}^{\mathrm{e}}$ siècle ; peut-être plus à l'ouest, en dehors de l'emprise de fouille. Peut-être enfin, est-ce ce très grand bâtiment, qui marque la limite sud du village. En effet, au moment de la Révolution française, la commune a son aspect actuel de village-rue. La maison des chanoines est située en bordure du village et sera l'une des victimes des fureurs anticléricales : vendue comme Bien national, elle sera démantelée rapidement, avant $1809^{8}$.
8. Le cadastre dit napoléonien, daté de 1809 , représente un terrain vierge de toute construction.

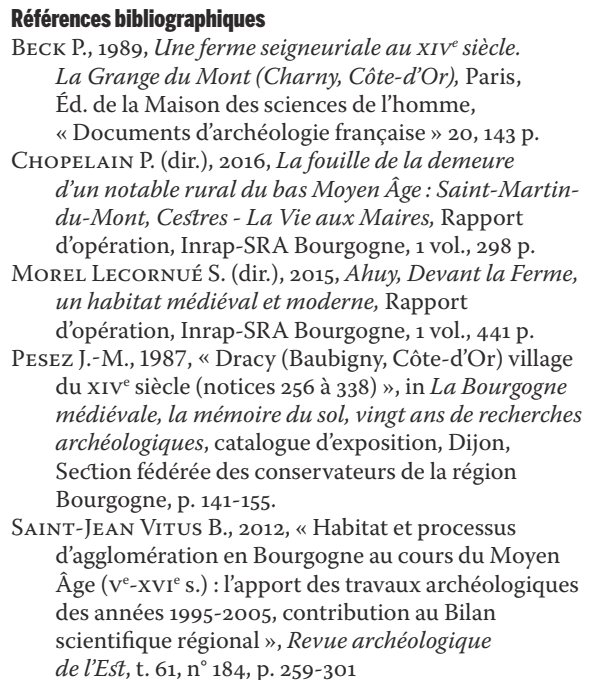

Références bibliographiques

P., 1989, Une ferme seigneu "Documents d'archéologie française » 20,143 p DEL AIN P. (dir), 2016, La fouille de la demeure d'un notable rural du bas Moyen Age : Saint-Martindu-Mont, Cestres - La Vie aux Maires, Rappor ération, Inrap-SRA Bourgogne, 1 vol., 298 p. un habitat médiéval et moderne, Rapport dopération, Inrap-SRA Bourgogne, 1 vol., 441 p. du XIV siècle (notices 256 à 338)", in La Bourgogne médiévale, la mémoire du sol, vingt ans de recherches archeologiques, catalogue d'exposition, Dijon, Bourgogne, p. 141-155 urgogne au cours du Moyen des années 1995-2005, contribution au Bilan de l'Est, t. $61, \mathrm{n}^{\circ} 184$, p. $259-30$ 